\begin{tabular}{|l|l|l||}
\hline \multicolumn{2}{|c|}{ PublisherInfo } \\
\hline \hline PublisherName & $:$ & BioMed Central \\
\hline \hline PublisherLocation & $:$ & London \\
\hline \hline PublisherImprintName & $:$ & BioMed Central \\
\hline \hline
\end{tabular}

\title{
Audit of deaths occurring after an ICU stay
}

\begin{tabular}{|l|l|l||}
\hline \multicolumn{2}{|c||}{ ArticleInfo } \\
\hline \hline ArticleID & $:$ & 4109 \\
\hline \hline ArticleDOI & $:$ & $10.1186 /$ ccf-1999-301 \\
\hline \hline ArticleCitationID & $:$ & 301 \\
\hline \hline ArticleSequenceNumber & $:$ & 46 \\
\hline \hline ArticleCategory & $:$ & Paper Report \\
\hline \hline ArticleFirstPage & $:$ & 1 \\
\hline \hline ArticleLastPage & $:$ & 4 \\
\hline \hline & & RegistrationDate : 1999-5-17 \\
\hline ArticleHistory & $:$ & OnlineDate \\
\hline \hline ArticleCopyright & $:$ & Current Science Ltd1999-5-17 \\
\hline \hline ArticleGrants & $:$ & \\
\hline \hline ArticleContext & $:$ & 130541111 \\
\hline \hline
\end{tabular}




\section{Keywords}

Complications, death, death in wards, intensive care

\section{Comments}

This is an interesting study and I think shows a surprisingly good ICU mortality of only $9 \%$ for patients in Waikato Hospital, New Zealand. Of 5916 patients studied, only five died unexpectedly on the ward and we are told that two of these were certainly unavoidable. These numbers are clearly a very small percentage but each death is a tragedy for the families and it would have been interesting to have more detailed data on the causes of death so that lessons could perhaps be learned.

\section{Introduction}

Admission to an Intensive Care Unit (ICU) is expensive and it is important to be clear that the benefit derived by each patient justifies the considerable use of resources. When a patient survives an intensive care admission but subsequently dies prior to hospital discharge, questions may be prompted about both care and treatment received on the main ward, and suitability of the patient for intensive care treatment.

\section{Aims}

This study set out to audit in-hospital deaths occurring followingdischarge from the ICU at Waikato Hospital, New Zealand. Particular aspects of interest were whether or not death was a likely outcome and if it could have been prevented.

\section{Methods}

Admissions between July 1991 and 1997 were examined, and these were divided into three groups; ICU deaths, ward deaths and hospital survivors. The ward death group was subdivided further into two 
groups: those 'expected to die' (Group 1) and those 'not expected to die' (Group 2). Group 2 patients were categorised in two further groups with regard to whether life expectancy was less than or more than one year and called 'likely to die' and 'not likely to die' respectively. Patients in Group 2 were studied further, and information collected concerning age, diagnosis, course in intensive care, premorbid history, number of systems failed, duration of ward stay and cause of death.

\section{Results}

A total of 5916 individual patients were admitted for ICU care during the 7 years studied, some requiring more than one admission. Of these, 5283 (89\%) survived, 525 (9\%) died in ICU and 108 (2\%) died on the ward. Data were only available on 99 of the ward death patients. $60(60 \%)$ deaths were expected (Group 1); $25 \%$ of these were cases of hypoxic cerebral damage with cerebrovascular accidents, and with renal, cardiac and respiratory failures as other major causes. Five (5\%) deaths were not likely or expected (Group2).No group 2 patient died from the disease process that prompted ICU admission; cardiac arrest being the main cause of death in these patients.One patient died of a previously undiagnosed, but inoperable dissecting aneurysm and another developed leukaemia. A total of 34 (34\%) deaths were in the 'likely to die ' group. Again, cardiac arrest was the main cause of death, with respiratory and renal failure being other causes. Six patients had multiple organ failure; pulmonary and cardiac disease being most common.

Twenty two of the 99 patients had been admitted to the ICU from the ward. Analysis of data from these patients showed that 11 were from the 'expected to die ' subgroup. Four of these patients had suffered avoidable problems prior to ICU admission. These included hypovolaemia resulting in renal failure, fluid overload leading to pulmonary oedema, delay in starting antibiotics despite proven infection, drug omission and inappropriate arrhythmia management. The remaining 11 patients were from the 'not expected to die' and 'likely to die' sub-groups. Four of these had also suffered avoidable complications. These were similar, and involved delay in taking blood cultures, inappropriate arrhythmia control, poor blood pressure control resulting in cardiac arrest and prolonged hypoxia resulting in respiratory arrest.

\section{Discussion}

Most deaths occurring after ICU discharge were due to continuing organ failure, rather than the step down to the less intensive level of care on the ward. This is in contrast to other published studies. The majority came from the 'expected to die' group in whom there was no longer a reversible or treatable element to the disease. Most of the other deaths were from patients whom were not expected to live for more than a year and it is not surprising than some, therefore, did not leave hospital. At least two of the five unexpected deaths were due to coincidental disease processes. Some of those patients dying with multisystem failure on the ward should perhaps not have been admitted to the ICU initially. Whilst triage of patients for ICU care is important, it is often a difficult decision and patients will often be given the benefit of the doubt. Management errors on the ward had clearly led to ICU admission in some 
cases, and, therefore may indirectly have resulted in deaths, but there was no recorded case of this occurring directly.

\section{References}

1. Lawrence A, Havill JH: An audit of deaths occurring in hospital after discharge from the intensive care unit. Anaesth Intens Care. 1999, 27: 185-189.

This PDF file was created after publication. 